Mindestbeteiligung von Frauen in Vorständen

\section{Sinnvolle und überfällige Maßnahme}

Damit haben viele nicht mehr gerechnet: Im Herbst 2020 einigten sich SPD und CDU auf einen Gesetzesentwurf für eine Mindestbeteiligung von Frauen in Vorständen, Anfang Januar hat das Bundeskabinett diesen Entwurf beschlossen. Verabschiedet der Bundestag im Laufe des Jahres den entsprechenden Gesetzesentwurf, müssten börsennotierte und zugleich paritätisch mitbestimmte Unternehmen, deren Vorstand vier oder mehr Mitglieder hat, in Zukunft mindestens einen Vorstandsposten mit einer Frau besetzen. Somit wären dann - sechs Jahre nach Einführung der Geschlechterquote für Aufsichtsräte - auch die Vorstände gesetzlich verpflichtet, unter bestimmten Voraussetzungen Frauen in ihre Reihen aufzunehmen.

Ist solch eine Mindestbeteiligung von Frauen in Vorständen eine sinnvolle Maßnahme? Die Antwort lautet ja. Natürlich wäre es schöner, wenn man keine staatlichen Vorgaben bräuchte, doch zahlreiche Analysen zeigen: Ohne gesetzliche Regelungen tut sich wenig. Das zeigt beispielsweise eine Analyse des Frauenanteils in Aufsichtsräten. Seit Einführung der Geschlechterquote 2015 ist ein starker Anstieg zu sehen. Unternehmen, die paritätisch mitbestimmt und börsennotiert sind und somit die Geschlechterquote für den Aufsichtsrat erfüllen müssen, haben den Frauenanteil in diesem Gremium nicht nur deutlich schneller erhöht als von der Größe her vergleichbare Unternehmen, die die Quote nicht erfüllen müssen, sondern sie haben den Frauenanteil in ihren Kontrollgremien auch über die vorgeschriebenen $30 \%$ hinaus gesteigert. In diesen "Quotenunternehmen“ liegt der Frauenanteil im Aufsichtsrat mittlerweile bei $36 \%$. Auch im internationalen Vergleich zeigt sich, dass da, wo es gesetzliche Vorgaben bezüglich des Frauenanteils gibt, die Entwicklung wesentlich dynamischer verläuft als da, wo es diese nicht gibt (Arndt und Wrohlich, 2019).

Eine mit der Quote für Aufsichtsräte verbundene Hoffnung war, dass damit in weiterer Folge auch der Frauenanteil im Vorstand steigen würde, quasi über eine Art „TrickleDown"-Effekt von Aufsichtsräten in Vorstände. Kurzfristig konnte ein solcher Effekt nicht nachgewiesen werden. Erst seit 2019 ist zu beobachten, dass der Frauenanteil in den Vorständen der Quotenunternehmen erkennbar stärker angestiegen ist als in von der Größe her vergleichbaren Unternehmen. Aber: Dieser Anstieg ist gering, der Frauenanteil bewegt sich in Vorständen noch immer auf äußerst niedri- gem Niveau. Selbst in der Gruppe der Quotenunternehmen betrug er im Herbst 2020 nur knapp 13\%. Eine Simulation zeigt, dass die vom Bundeskabinett beschlossene Mindestbeteiligung den Frauenanteil in den betroffenen Unternehmen von derzeit rund $13 \%$ auf $21 \%$ steigern würde, sobald alle die Vorgabe erfüllen (Kirsch und Wrohlich, 2021).

Von einer gesetzlichen Regelung für die Vorstände sind zudem gleichstellungspolitische Auswirkungen zu erwarten, die über die reine Zahl von Frauen in Vorständen hinausgehen. Da Mitglieder des Vorstands - im Vergleich zu Aufsichtsratsmitgliedern - weniger heterogene berufliche Hintergründe haben und oft eine unternehmensinterne Karriere durchlaufen, wären die Unternehmen durch solche gesetzlichen Vorgaben gezwungen, stärker auf ihre Führungskräfteentwicklung zu achten. Insbesondere müssten sie darauf hinarbeiten, dass genügend Frauen unternehmensintern befördert werden, um sich für Vorstandspositionen zu qualifizieren. Ein höherer Frauenanteil auf allen Managementebenen könnte wiederum gleichstellungspolitische Wirkungen auf das gesamte Unternehmen entfalten. Beispielsweise gibt es empirische Belege dafür, dass ein höherer Anteil von Frauen in Führungspositionen in einem Unternehmen geringere Gender Pay Gaps, also geringere Verdienstunterschiede zwischen Frauen und Männern in diesem Unternehmen zur Folge haben und die Chancen für eine Beförderung von Frauen steigen.

Zudem zeigt experimentelle Forschung, dass Frauen auf dem Arbeitsmarkt aufgrund geschlechterstereotyper Zuschreibungen vielerorts nach wie vor nicht die gleichen Chancen haben wie Männer. Eine Geschlechterquote oder Mindestbeteiligung kann das in mehrfacher Weise ändern. Zum einen wird das unter dem Stichwort „Thomas-Kreislauf" bekannt gewordene Phänomen ausgehebelt, dass homogene Gruppen - beispielsweise Vorstände in groBen deutschen Unternehmen - dazu neigen, sich selbst zu reproduzieren. Die Vorstände wären dann gezwungen, vor allem Bewerberinnen mit teilweise wohl ganz anderen Hintergründen genauer in Betracht zu ziehen. Zum anderen können mehr Frauen in hohen Führungspositionen dazu beitragen, dass geschlechterstereotype Zuschreibungen in der gesamten Gesellschaft sukzessive verringert werden.

\section{Literatur}

Arndt, P. und K. Wrohlich (2019), Geschlechterquoten im europäischen Vergleich: Harte Sanktionen bei Nichteinhaltung sind am wirkungsvollsten, DIW Wochenbericht, 38, 691-698.

Kirsch, A. und K. Wrohlich (2021), Mehr Frauen in Spitzengremien großer Unternehmen, Dynamik aber verhalten, DIW Wochenbericht, 3, 22-34. (c) Der/die Autor:in(nen) 2021. Open Access: Dieser Artikel wird unter der Creative Commons Namensnennung 4.0 International Lizenz veröffentlicht (creativecommons.org/licenses/by/4.0/deed.de).

Open Access wird durch die ZBW - Leibniz-Informationszentrum Wirtschaft gefördert.
Katharina Wrohlich Deutsches Institut für Wirtschaftsforschung (DIW Berlin) kwrohlich@diw.de 